\title{
Capture coefficients of free excitons by shallow acceptors and donors in gallium arsenide
}

\author{
K.D. Glinchuk, N.M. Litovchenko, O.N. Strilchuk \\ Lashkaryov Institute of Semiconductor Physics, NAS of Ukraine, 45 prospect Nauky, 03028 Kyiv, Ukraine \\ Phone : +38(044) 265 6373; fax: +38(044) 2653337; e-mail: strilchuk@isp.kiev.ua
}

\begin{abstract}
An analysis of the $4.2 \mathrm{~K}$ exciton luminescence spectra of semi-insulating GaAs crystals with different concentrations of shallow acceptors $(\mathrm{C})$ and donors $(\mathrm{Si})$ is given. As a result, the $4.2 \mathrm{~K}$ capture coefficients of free excitons by shallow neutral acceptors $\left[b_{A^{0} X}=\right.$ $\left.=(4 \pm 2) \cdot 10^{-8} \mathrm{~cm}^{3} / \mathrm{s}\right]$ and donors $\left[b_{D^{0} X}=(1.5 \pm 0.8) \cdot 10^{-7} \mathrm{~cm}^{3} / \mathrm{s}\right]$ are found and also an estimate of the capture coefficient of free excitons by ionized shallow donors was made $\left[b_{D^{+} X}>>b_{D^{0} X}\right]$.
\end{abstract}

Keywords: gallium arsenide, shallow acceptors and donors, exciton luminescence, capture coefficients of free excitons.

Paper received 09.06.03; accepted for publication 16.06.03.

\section{Introduction}

As is known, in semiconductors effective processes of binding free excitons (their concentration $n_{X}$ ) by shallow neutral acceptors $A^{0}$, shallow ionized $D^{+}$and neutral $D^{0}$ donors (further neutral acceptors, ionized and neutral donors, their concentrations accordingly $N_{A^{0}}, N_{D^{+}}$and $N_{D^{0}}$ ) take place [these processes lead to formation of bound exciton-neutral acceptor $\left(A^{0} X\right)$, bound excitonionized donor $\left(D^{+} X\right)$ and bound exciton - neutral donor $\left(D^{0} X\right)$ complexes]. The important characteristics of the rate of above-pointed binding processes are capture coefficients of free excitons by neutral acceptors $b_{A^{0} X}$, ionized $b_{D^{+} X}$ and neutral $b_{D^{0} X}$ donors. But in the literature practically no data about $b_{A^{0} X}, b_{D^{+} X}$ and $b_{D^{0} X}$ values in semiconductors exist. Really, only in [1] a theoretical examination of free exciton binding by neutral impurities in semiconductors was carried out (unfortunately, the expression for $b_{A^{0} X}$ is not given in the cited paper), and in $[2,3]$ an attempt to evaluate the free exciton capture coefficient by shallow neutral and ionized traps in CdS was made $\left(b_{A^{0} X}=3 \cdot 10^{-10} \mathrm{~cm}^{3} / \mathrm{s}\right.$ or $3 \cdot 10^{-7} \mathrm{~cm}^{3} / \mathrm{s}$ ac- cording to [2], and $b_{D^{+} X}=6 \cdot 10^{-8} \mathrm{~cm}^{3} / \mathrm{s}, b_{D^{0} X}=$ $=6 \cdot 10^{-9} \mathrm{~cm}^{3} / \mathrm{s}$ according to [3]). In the present work, as a result of the analysis of the excitonic luminescence spectra of semi-insulating GaAs crystals at $T=4.2 \mathrm{~K}$ (at low temperatures they are photoconductors [4]) with different concentrations of shallow acceptors $N_{A}$ and donors $N_{D}$, we will give the $4.2 \mathrm{~K} b_{A^{0} X}$ and $b_{D^{0} X}$ values and evaluate the $4.2 \mathrm{~K} b_{D^{+} X}$ value for gallium arsenide.

\section{Method for obtaining $b_{A^{0} X}, b_{D^{+} X}$ and $b_{D^{0} X}$ values}

A method to obtain the $b_{A^{0} X}, b_{D^{+} X}$ and $b_{D^{0} X}$ values follows from an analysis of intensities of excitonic emission bands in photoconductors [their conductivity is determined by photoelectrons (concentration $\delta n$ ) and photoholes ( concentration $\delta p$ ), in them at $T=4.2 \mathrm{~K}$ $N_{A^{0}}=N_{D^{0}}=0, N_{D^{+}}=N_{D}$ in darkness and $N_{A^{0}} \approx N_{A}$, $N_{D^{0}} \approx N_{D}>N_{D^{+}}$when illuminated (the given $N_{A^{0}}$, $N_{D^{+}}$and $N_{D^{0}}$ values in illuminated photoconductors are correct for isolated acceptors and donors [4], they predominantly bind free excitons in semi-insulating gallium 


\section{K.D. Glinchuk et al.: Capture coefficients of free excitons ...}

arsenide [5])]. Evidently, in photoconductors luminescence intensities, induced by annihilation of exciton-impurity complexes $A^{0} X\left(I_{A^{0} X}\right), D^{+} X\left(I_{D^{+} X}\right)$ and $D^{0} X\left(I_{D^{0} X}\right)$ and also by recombination of free excitons $X\left(I_{X}\right)$, are given by the following relations \{when writing Eqs (1)(4) we assumed that, firstly, the concentrations of acceptors and donors that bound excitons is small, and secondly, annihilation of free and bound excitons proceeds mainly with the photon emission [4]:

$$
\begin{aligned}
& I_{A^{0} X}=b_{A^{0} X} N_{A^{0} n_{X}} \approx b_{A^{0} X} N_{A^{n}} n_{X}, \\
& I_{D^{+} X}=b_{D^{+} X} N_{D^{+}} n_{X}, \\
& I_{D^{0} X}=b_{D^{0} X} N_{D^{0}} n_{X} \approx b_{D^{0} X} N_{D^{n}} n_{X}, \\
& I_{X}=\alpha_{X} n_{X},
\end{aligned}
$$

where $\alpha_{X}$ is the probability of the free exciton radiative annihilation the $4.2 \mathrm{~K} \alpha_{X}$ value is $\alpha_{X}=2.5 \cdot 10^{8} \mathrm{~s}^{-1}$, as follows from the $4.2 \mathrm{~K}$ decay time of the free excitoninduced luminescence $\tau_{X}=1 / \alpha_{X} \approx 4$ ns [6]; note here that if at low temperatures free excitons have the Maxwellian distribution, then theoretically $\alpha_{X}=3 \cdot 10^{9} \mathrm{~s}^{-1}$ [7]; a difference in experimental and theoretical $\alpha_{X}$ values testifies that the distribution of free excitons at low temperatures is not the Maxwellian one . So, as one could see from Eqs (1)-(4), the $4.2 \mathrm{~K} b_{A^{0} X}, b_{D^{+} X}$ and $b_{D^{0} X}$ values could be found from the following obvious relations:

$$
\begin{aligned}
& b_{A^{0} X}=\frac{I_{A^{0} X}}{I_{X}} \frac{\alpha_{X}}{N_{A}}, \quad b_{D^{+} X}=\frac{I_{D^{+} X}}{I_{X}} \frac{\alpha_{X}}{N_{D^{+}}}, \\
& b_{D^{0} X}=\frac{I_{D^{0} X}}{I_{X}} \frac{\alpha_{X}}{N_{D}},
\end{aligned}
$$

if we use the available experimental $I_{A^{0} X} / I_{X}, I_{D^{+} X} / I_{X}$, $I_{D^{0} X} / I_{X}, N_{A}, N_{D^{+}}$and $N_{D}$ values and the known $\alpha_{X}$ value (see Appendix).

\section{Experimental}

In our experiments, semi-insulating GaAs crystals were used (the specific resistivity $\rho \approx 4 \cdot 10^{7} \mathrm{Ohm} \cdot \mathrm{cm}$ at $300 \mathrm{~K}$ and $\rho \rightarrow \infty$ at $T=4.2 \mathrm{~K}$ ). Their dark $300 \mathrm{~K}$ conductivity was determined by ionization of deep native donor defects EL2 (concentration $N_{E L 2} \approx 2 \cdot 10^{16} \mathrm{~cm}^{-3}$ ) partly compensated by shallow acceptors (carbon atoms, concentration $N_{A}$ ) and donors (silicon atoms, concentration $N_{D}$ ), i.e. $N_{E L 2}>N_{A}-N_{D}>0$ [8] \{the concentration of $E L 2$ defects and equilibrium room-temperature concentration of ionized $E L 2$ defects $E L 2^{+} N_{E L 2}^{+}=N_{A}-$
$N_{D}>0$ were found (with the accuracy $\pm 10 \%$ ) from $300 \mathrm{~K}$ infrared absorption spectrum associated with the deep donor EL2 [8-10]\}. When excited (at $T=4.2 \mathrm{~K}$ ) their conductivity was determined by excess electrons and holes [5,8]. The 4.2 K luminescence spectra (excitation intensity $L$ ) of semi-insulating GaAs crystals with different concentrations of carbon and silicon were studied the carbon concentration (with the accuracy $\pm 20 \%$ ) was found from the usual $77 \mathrm{~K}$ infrared absorption measurements at $\lambda=17.2 \mu \mathrm{m}$ (wavenumber $580 \mathrm{~cm}^{-1}$ ); the silicon concentration (with the accuracy $\pm 30 \%$ ) was estimated from the mass - spectroscopy analysis and the known equilibrium concentration of positively charged $E L 2$ defects $N_{E L 2}^{+}$and carbon content: obviously $N_{D}=N_{A}-$ $-N_{E L 2}^{+}$at $T=300 \mathrm{~K}$ as $N_{D^{+}} \approx N_{D}$ and $N_{A^{0}}=0$ at room temperature $[4,8]\}$. In the $4.2 \mathrm{~K} \mathrm{GaAs}$ luminescence spectrum (after its decomposition by individual components with the accuracy $\pm 20 \%$ ), emission bands induced by annihilation of bound excitons $A^{0} X$ (peak position $\left.h v_{m}=1.512 \mathrm{eV}\right), D^{+} X \quad\left(h v_{m}=1.5133 \mathrm{eV}\right)$ and $D^{0} X$ $\left(h v_{m}=1.5141 \mathrm{eV}\right)$ and also by recombination of free excitons $X\left(h v_{m}=1.5153 \mathrm{eV}\right)$ were clearly seen (Fig. 1) [4]. The emission intensities $I_{A^{0} X}, I_{D^{+} X}, I_{D^{0} X}$ and $I_{X}$ as functions of carbon and silicon concentration were found. At low excitation powers, the used intensities $I_{A^{0} X}, I_{D^{+} X}$, $I_{D^{0} X}$ and $I_{X}$ changed quadratically with $L\left(I_{A^{0} X}\right.$, $I_{D^{+} X}, I_{D^{0} X}, I_{X} \sim L^{2}$, see [4,5]); the same $I_{A^{0} X}, I_{D^{+} X}^{A_{X}}$, $I_{D^{0} X}, I_{X}$ vs. $L$ dependencies testify that in crystals studied $N_{A^{0}}, N_{D^{+}}, N_{D^{0}} \neq \varphi(L)$ [4]. As expected (see [4]) normalized bound exciton $A^{0} X$ induced emission intensity $I_{A^{0} X} / I_{X}$ changed linearly with $N_{A}$ and normalized bound exciton $D^{0} X$ induced emission intensity $I_{D^{0} X} / I_{X}$ changed linearly with $N_{D}$ i.e. $I_{A^{0} X} / I_{X} \sim N_{A}$ and $I_{D^{0} X} / I_{X} \sim N_{D}$ (see Fig. 2).

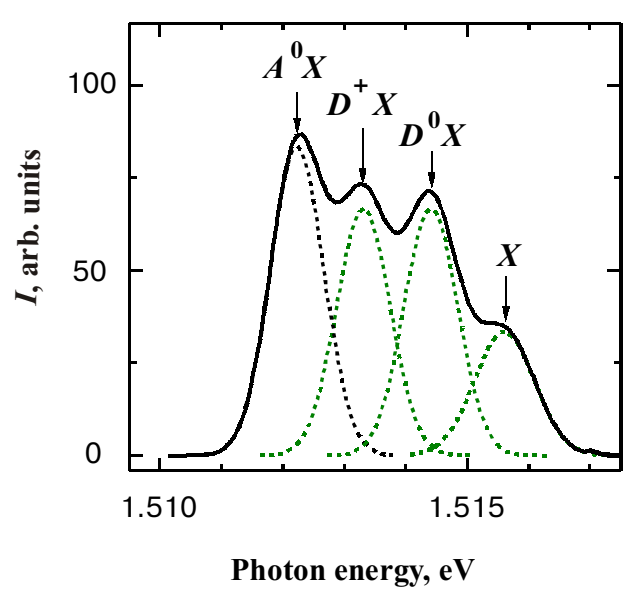

Fig. 1. The $4.2 \mathrm{~K}$ bound $\left(A^{0} X, D^{+} X, D^{0} X\right)$ and free $(X)$ excitoninduced emission bands in the luminescence spectrum of semiinsulating GaAs crystals. 


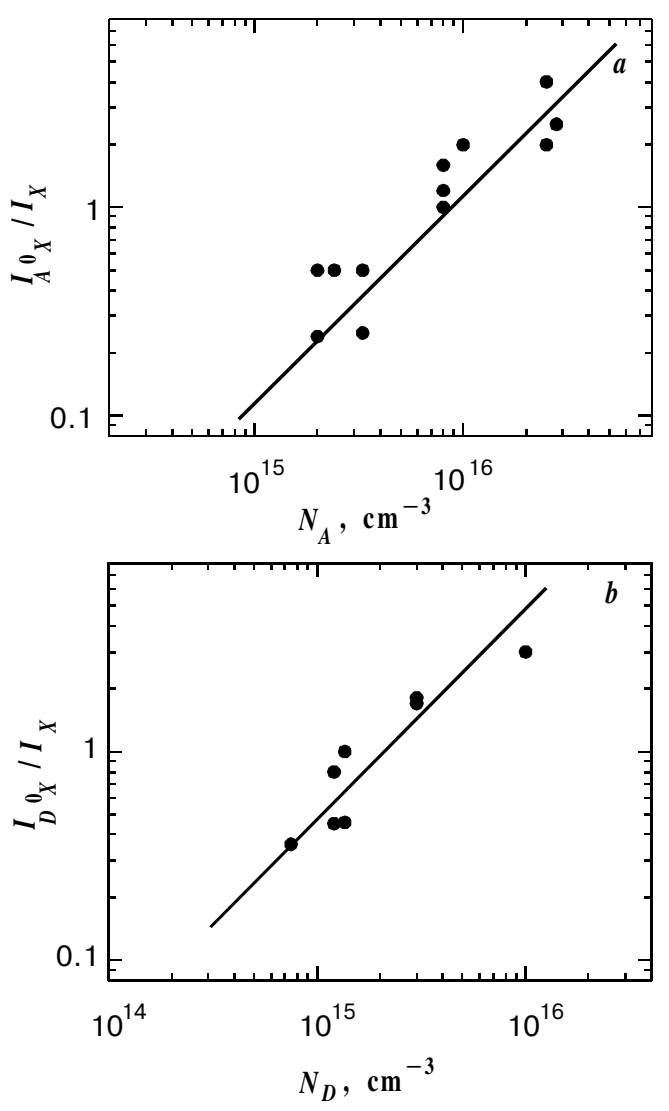

Fig. 2. $I_{A^{0} X} / I_{X}$ vs. $N_{A}(a)$ and $I_{D^{0} X} / I_{X}$ vs. $N_{D}(b)$ variations in semi-insulating GaAs at $T=4.2 \mathrm{~K}$. The straight lines - theoretical dependences [they follow from Eqs (1), (3) and (4)] $I_{A^{0} X} / I_{X}=$ $=\left(b_{A^{0} X} / \alpha_{X}\right) N_{A}(a)$ and $I_{D^{0} X} / I_{X}=\left(b_{D^{0} X} / \alpha_{X}\right) N_{D}(b)$, where $b_{A^{0} X}=4 \cdot 10^{-8} \mathrm{~cm}^{3} / \mathrm{s}, b_{D^{0} X}=1.5 \cdot 10^{-7} \mathrm{~cm}^{3} / \mathrm{s}$ and $\alpha_{X}=3 \cdot 10^{8} \mathrm{~s}^{-1}$. A deflection of experimental points from the theoretical straight lines shows the accuracy of $b_{A^{0} X}$ and $b_{D^{0} X}$ determination.

\section{Results and discussion}

The following $4.2 \mathrm{~K}$ values for the capture coefficients of free excitons by neutral acceptors and donors were found [see Eq. (5) and also Fig. 2]: $b_{A^{0} X}=(4 \pm 2) \cdot 10^{-8} \mathrm{~cm}^{3} / \mathrm{s}$ and $b_{D^{0} X}=(1.5 \pm 0.8) \cdot 10^{-7} \mathrm{~cm}^{3} / \mathrm{s}$ [rather small accuracy of $b_{A^{0} X}$ and $b_{D^{0} X}$ measurements are connected with a fairly large inaccuracies in the $N_{A}$ and $N_{D}$ determination and in the decomposition of GaAs luminescence spectrum on individual $A^{0} X, D^{0} X$ and $X$ components (i.e. when determining of $I_{A^{0} X}, I_{D^{0} X}$ and $I_{X}$ values)]. As the $4.2 \mathrm{~K}$ $N_{D^{+}}$value is unknown (obviously, $N_{D^{+}} \ll<N_{D}$ ), we could only evaluate the $b_{D^{+} X}$ value $-b_{D^{+} X}>>b_{D^{0} X}$ (this follows from the usually observed in an experiment relation $\left.I_{D^{+} X} / I_{D^{0} X}=b_{D^{+} X} N_{D^{+}} / b_{D^{0} X} N_{D^{0}} \leq 1\right)$. So, in gallium arsenide, the following relation between $b_{A^{0} X}, b_{D^{+} X}$ and $b_{D^{0} X}$ exists: $b_{A^{0} X}<b_{D^{0} X} \ll b_{D^{+} X}$. The found correlation between $b_{A^{0} X}, b_{D^{+} X}$ and $b_{D^{0} X}$ values could be explained as follows. A formation of bound excitons $A^{0} X, D^{+} X$ and $D^{0} X$ occurs due to attraction of free excitons to acceptors and donors as a result of dipoledipole (this refers to $X$ and $A^{0}, X$ and $D^{0}$ ) or dipolecharge (this refers to $X-D^{+}$) interaction (obviously, dipoles are induced by the mutual polarization of excitons and neutral acceptors, donors). Undoubtedly, a dipole momentum of neutral acceptors is less than that of neutral donors due to a rather small Böhr radius of the acceptor-bound hole compared to that of donor-bound electron and a dipole momentum of ionized donors exceeds that of neutral donors. As a result, one expects that the relations $b_{A^{0} X}<b_{D^{0} X}<<b_{D^{+} X}$ will be observed in semiconductors.

The obtained $4.2 \mathrm{~K} b_{A^{0} X}, b_{D^{+} X}$ and $b_{D^{0} X}$ values in $\mathrm{GaAs}$ appear to be rather large, so they illustrate a relatively high efficiency of free exciton trapping by shallow acceptors and donors in gallium arsenide.

\section{Conclusions}

The $4.2 \mathrm{~K}$ capture coefficients of free excitons by neutral acceptors and ionized and neutral donors for gallium arsenide were found and analyzed. The obtained data are important for understanding of regularities of the bound exciton induced luminescence in intermetallic semiconductors, which are widely studied now.

\section{Appendix}

Evidently, Eqs (1) - (4) used as a basis for obtaining experimental $b_{A^{0} X}, b_{D^{+} X}$ and $b_{D^{0} X}$ values [see Eq.(5)] are valid for uniformly excited photoconductors [in them $\delta n, \delta p \neq \varphi(y)$, where $y$ is the propagation direction of the exciting light]. Obviously, if excitation is non-uniform [i.e. $\delta n, \delta p=\varphi(y)]$ then the excitonic emission intensities from a photoconductor are:

$I_{A^{0} X}=b_{A^{0} X} \int N_{A^{0}} n_{X} d y \approx b_{A^{0} X} N_{A} \int n_{X} d y$,

$I_{D^{+} X}=b_{D^{+} X} \int N_{D^{+}} n_{X} d y$,

$I_{D^{0} X}=b_{D^{0} X} \int N_{D^{0}} n_{X} d y \approx b_{D^{0} X} N_{D} \int n_{X} d y$,

$I_{X}=\alpha_{X} \int n_{X} d y$,

where integration is over the extent of the light-emitting region in the $y$-direction (in this region, as was pointed above, $N_{A^{0}} \approx N_{A}$ and $\left.N_{D^{0}} \approx N_{D}>>N_{D^{+}}\right)$.

So, as one could see from Eqs (A1)-(A4), in a discussed case (non-uniform excitation of photoelectrons and photoholes) the $b_{A^{0} X}, b_{D^{+} X}$ if $\delta n / \delta p \neq \varphi(y)$, then $N_{D^{+}} \neq$ 


\section{K.D. Glinchuk et al.: Capture coefficients of free excitons ...}

$\neq \varphi(y)$ in a light-emitting region, see [4] $\}$, and $b_{D^{0} X}$ values could also be found from Eq. (5). From this follows that the proposed method of determination of $b_{A^{0} X}$, $b_{D^{+} X}$ and $b_{D^{0} X}$ values [from Eq. (5)] could be used for any kind (uniform or non-uniform) photoconductor excitation.

\section{References}

1. A.A. Lipnic. Bounding and dissociation of Motts excitons on phonons and impurity centres // Fiz. tverd. tela, 3(8), pp. 23222330 (1961) (in Russian).

2. D. Magde, H. Mahr, Kinetics of excitons in CdS at low temperature // Phys.Rev.B., 2(10), pp.4098-4103 (1970)

3. V.L. Broude, I.I. Tartakovsky, V.B. Timofeev, Kinetics of luminescence of free and bound excitons in CdS crystals // Fiz. tverdogo tela, 14(12), pp. 3531-3539 (1972) (in Russian).

4. K.D. Glinchuk, A.V.Prokhorovich, Pecularities of determination of the shallow-level impurity concentrations in semiconductors from the analysis of the exciton luminescence spectrum // Fiz.Tekhnika Poluprov, 36(5), pp. 519-524 (2002) (in Russian).
5. K.D. Glinchuk, A.V. Prokhorovich, Analysis of the nearband-edge luminescence of semiconductors containing isolated and bound shallow acceptora and donors // Semiconductor Physics, Quantum electronics and Optoelectronics, 5(4), pp. 353-361 (2002).

6. G.W. Hooft, W.A. Poel, L.W. Molenkamp, C.T. Foxon, Giant oscillator strength of free excitons in GaAs // Phys.Rev.B., 35(15), pp. 8281-8284 (1987).

7. A.P. Levanyuk, V.V. Osipov, Edge luminescence of the direct-gap semiconductors // Uspekhi fiz. nauk., 133(3), pp. 427-477 (1981) (in Russian).

8. K.D. Glinchuk, Y.I. Guroshev, A.V. Prokhorovich, Use of photo- and cathodoluminescence for studying of the physical properties of semi - insulating undoped GaAs crystals (a review) // Optoelectronics and Semiconductor technique., 24, pp. 66-96 (1992) (in Russian).

9. K. Brierly, D.S. Lehr, Full wafer-mapping of total and ionized EL2 concentration in semi-insulating GaAs using infrared absorption // Appl.Phys.Lett., 55(23), pp.2426 -2428 (1989).

10. M. Suemitsu, K. Terada, M. Nishiima, N. Miyamoto, Mechanism for homogenizing electrical properties of semiinsulating GaAs during annealing // J.Appl.Phys., 70(5), pp. 2594-2598 (1991). 